\title{
PROPERTIES OF NEUTRON STARS IN THE RELATIVISTIC MEAN-FIELD THEORY
}

\author{
K. S. Cheng, ${ }^{1,2}$ Z. G. DAI, ${ }^{3}$ AND C. C. YAO $^{1}$ \\ Received 1995 September 18; accepted 1995 December 22
}

\begin{abstract}
We study the properties of dense matter in neutron stars and calculate the structure of the stars based on the Zimanyi \& Moszkowski (ZM) model in the relativistic mean-field theory. We also compare these results with those based on the Boguta \& Bodmer (BB) model with a recent satisfactory parameter set. The two models satisfy the requirements from the observations of the masses of binary radio pulsars, the rotation frequencies of millisecond pulsars, the redshifts of the $e^{+}$annihilation lines of some $\gamma$-ray bursts if they are neutron stars, and the crustal moment of inertia of neutron stars deduced from the glitch events. Other observations may provide a way to discriminate between the two models. We suggest that the most important observational discriminant between these two models is found by observing the surface radiation of neutron stars, since the BB model leads to a large photon fraction of neutron star matter and rapid cooling of neutron stars, but the ZM model does not.
\end{abstract}

Subject headings: dense matter - pulsars: general - relativity - stars: interiors - stars: neutron

\section{INTRODUCTION}

Usually, after knowing an equation of state (EOS) for dense matter, one can calculate neutron star properties such as the mass range, the mass-radius relationship, and the moment of inertia, and one can determine the composition of the matter and the thickness of the crust in a neutron star. These theoretical results can be compared with astronomical observations. First, the observations of binary radio pulsars perhaps give neutron star masses of $1.4 M_{\odot}<$ $M_{\max }<1.85 M_{\odot}$ (Taylor \& Weisberg 1989; Joss \& Rappaport 1984), which may be used to constrain the EOS of a neutron star. Second, the discovery of the $1.56 \mathrm{~ms}$ pulsar PSR $1937+21$ (Backer et al. 1982) initiated the investigation of a growing group of millisecond pulsars (with $P<10 \mathrm{~ms}$ ). Up to now, more than 30 millisecond pulsars have been detected (Taylor, Manchester, \& Lyne 1993). These observations have stimulated the study of the maximum rotation frequency, which is determined by the maximum mass of a neutron star and the radius of the star (Friedman, Ipser, \& Parker 1989; Lattimer et al. 1990; Cook, Shapiro, \& Teukolsky 1994). Third, one has seen glitch events of about 20 pulsars. According to the neutronsuperfluid vortex creep theory (Alpar et al. 1984), the postglitch recovery in a pulsar is largely dependent upon the ratio of the moment of inertia for the inner crust neutron superfluid of the star to the total moment of inertia. Fourth, the surface gravitational redshifts of neutron stars have been observed from $e^{+} / e^{-}$annihilation lines of Her X-1 (Trümper et al. 1978) and possibly some gamma-ray bursts (Harding 1991). Theoretical redshifts can be calculated directly through the masses and radii of neutron stars. Finally, the Einstein, EXOSAT, and ROSAT satellites have detected the thermal radiation from the surfaces of some neutron stars (Ögelman 1995). Theoretically, one can study the thermal evolution of a neutron star by giving an EOS in the star's interior (Tsuruta 1995, and references therein). The comparison between observed and theoretical results

\footnotetext{
${ }^{1}$ Department of Physics, University of Hong Kong, Pokfulam Road, Hong Kong.

2 hrspksc@hkucc.hku.hk.

${ }^{3}$ Department of Astronomy, Nanjing University, Nanjing 210093, China.
}

may set some limits on an EOS for dense matter. Hence, the determination of an EOS for dense matter is crucial in studying the physics of neutron stars.

Among the many approaches (for a brief review, see Prakash, Ainsworth, \& Lattimer 1988) to determine an EOS for dense matter through the many-body theory of interacting hadrons, the relativistic many-body approach to nuclear systems is of growing interest during recent years. The relativistic Brueckner-Hartree-Fock theory reproduces the saturation property of nuclear matter, which is not possible in the nonrelativistic approach unless one introduces three-body force by hand (Brockmann \& Machleidt 1990). Moreover, rather promising results within the framework of this theory have been obtained by Müther, Machleidt, \& Brockmann (1990) and Li, Machleidt, \& Brockmann (1992), and the application of this theory to neutron stars has been investigated by Engvik et al. (1994), Bao et al. (1994), and Sumiyoshi, Oyamatsu, \& Toki (1995b). On the other hand, the relativistic mean field (RMF) theory is successful both for elastic scattering and for nuclear ground-state property (Walecka 1974; Chin 1977; Serot \& Walecka 1986). Hence, the RMF theory has been suggested for calculating the EOS for neutron-star matter (Walecka 1974). This approach contains both nucleonic and mesonic degrees of freedom $(\sigma, w$, and $\rho$ ) and can be considered as phenomenological. The coupling constants and meson masses of the effective meson-nucleon Lagrangian are taken as free parameters which are adjusted to fit the properties of nuclear matter and finite nuclei.

In the standard model of Walecka (1974), the incompressibility of nuclear matter is overestimated. There are two ways to solve this question. First, Boguta \& Bodmer (1977, hereafter BB) introduced cubic and quartic terms for the scalar field into the Lagrangian. This shifts the incompressibility to reasonable values in comparison with empirical data. Along this direction, many authors (Glendenning 1982, 1985, 1987a, b; Weber \& Weigel 1989a, b; Kapusta \& Olive 1990; Ellis, Kapusta, \& Olive 1991; Sumiyoshi, Toki, \& Brockmann 1992; Sumiyoshi \& Toki 1994; Sumiyoshi, Kuwabara, \& Toki 1995a) have studied the EOS for dense matter and the properties of neutron stars. Zimanyi \& Moszkowski (1990, hereafter ZM) proposed an alternative nonlinear model, in which the nonlinearity is contained in 
the connection between the effective nucleon mass and the scalar field. Thus, the Lagrangian of this model has no extra terms, and consequently it deals with fewer parameters as compared with the BB model. The ZM model also yields reasonable values of incompressibility and an effective nucleon mass for nuclear matter. This model has been used recently to study the possibility of quark-hadron phase transition in proto-neutron stars (Prakash, Cooke, \& Lattimer 1995). The scope of our work is to derive the EOS for asymmetric nuclear matter and to study the properties of neutron stars, by using the ZM model. To our knowledge, this has not been done before. In addition, we will compare the results with those based on the BB model with a rather satisfactory parameter set. Note that the ZM and BB models are usually referred to as the nonlinear $\sigma \omega$ models.

It is well known that the applications for the nonlinear $\sigma \omega$ models to finite nuclei and semi-infinite matter are quite complicated on a purely quantum mechanical level, since the evaluation of energies and particle density distribution within the Hartree approximation demands the knowledge of wave functions of all occupied single-particle states. To avoid this task, Centelles et al. $(1992,1993)$ recently proposed the extended Thomas-Fermi approximation (ETF) extended up to second order in $\hbar$ based on a semiclassical method. In the ETF approximation, the total energy of a nuclear system is expressed as a function of the local particle density and its gradients. But for infinite nuclear matter (e.g., neutron star matter), the Hartree and ETF approximations turn out to be the same.

In this paper, we choose two recent sets of parameters named ZM and SRK3M7, which correspond to the ZM and BB models, respectively. These parameter sets have been shown to reproduce the experimental properties of many nuclei and to fit accepted nuclear matter data (e.g., Centelles et al. 1992). By using the nonlinear $\sigma \omega$ models to calculate the EOS for dense matter and to study the properties of neutron stars, we show that the extrapolation of these models to neutron star matter at high densities is rather different. We arrange this paper as follows. In $\S 2$, we describe the framework based on the nonlinear $\sigma \omega$ models, and we give the semiclassical energy densities. In $\S 3$, we calculate the properties of neutron stars and compare the results from the ZM model with those from the BB model. A detailed discussion of our results is presented in $\S 4$.

\section{NONLINEAR $\sigma \omega$ MODELS}

In the relativistic mean field theory, the strong interaction is described by the exchange of mesons between nucleons through the Yukawa couplings. But in the model of Walecka (1974), only $\sigma$ and $\omega$ mesons are included. In order to describe actual nuclear systems, it is necessary to introduce proton-neutron asymmetry effects. This is done by adding the $\rho$-meson contribution and the electromagnetic field. We follow the notation of Centelles et al. $(1992,1993)$. The Lagrangian density of the nuclear system is given by

$$
\begin{aligned}
\mathscr{L}= & \bar{\psi}\left[\gamma_{\mu}\left(i \partial^{\mu}-g_{v} V^{\mu}\right)-m^{*}\right] \psi \\
& +\frac{1}{2}\left(\partial_{\mu} \phi \partial^{\mu} \phi-m_{s}^{2} \phi^{2}\right) \\
& -\frac{1}{3} b \phi^{3}-\frac{1}{4} c \phi^{4}-\frac{1}{4} F_{\mu \nu} F^{\mu v} \\
& +\frac{1}{2} m_{v}^{2} V_{\mu} V^{\mu}-\frac{1}{4} H_{\mu \nu} H^{\mu v} \\
& -e \bar{\psi} \gamma_{\mu} \frac{1}{2}\left(1+\tau_{3}\right) A^{\mu} \psi-\frac{1}{4} \boldsymbol{G}_{\mu v} \cdot \boldsymbol{G}^{\mu v} \\
& +\frac{1}{2} m_{p}^{2} \boldsymbol{b}_{\mu} \cdot \boldsymbol{b}^{\mu}-\frac{1}{2} g_{\rho} \bar{\psi} \gamma_{\mu} \tau \cdot \boldsymbol{b}^{\mu} \psi
\end{aligned}
$$

where

$$
\begin{aligned}
F_{\mu \nu} & =\partial_{\mu} V_{v}-\partial_{v} V_{\mu}, \\
H_{\mu \nu} & =\partial_{\mu} A_{v}-\partial_{\nu} A_{\mu}, \\
G_{\mu \nu} & =\partial_{\mu} b_{v}-\partial_{v} b_{\mu} .
\end{aligned}
$$

Here $\psi, \phi, V^{\mu}$, and $\boldsymbol{b}^{\mu}$ denote the fields of the baryon, the attractive isoscalar-scalar $(\sigma)$ meson, the repulsive isoscalarvector $(\omega)$ meson, and the isovector-vector $(\rho)$ meson with masses of $m_{\sigma}, m_{\omega}$, and $m_{\rho}$, respectively. $A^{\mu}$ is the electromagnetic field. The constants $g_{\sigma}, g_{\omega}$, and $g_{\rho}$ are coupling constants for interactions between mesons and nucleons. The coefficients $b$ and $c$ are self-coupling constants for the $\sigma$ meson field. The $m^{*}$ is the effective nucleon mass. In the ZM model,

$$
b=c=0 \quad \text { and } \quad m^{*}=\frac{m}{1+g_{s} \phi / m} ;
$$

but in the BB model,

$$
b \neq 0, c \neq 0, \text { and } m^{*}=m-g_{s} \phi .
$$

Several authors (Glendenning 1985, Ellis et al. 1991) have studied the neutron star matter in the RMF theory with strangeness degrees of freedom and discussed the contamination of strange hadrons in neutron stars. The possibility of strange quark stars has been also discussed (e.g., Witten 1984; Alcock, Farhi, \& Olinto 1986; Haensel, Zdunik, \& Schaeffer 1986). The pion condensation and the kaon condensation in the RMF theory and their applications to neutron stars have been studied (Glendenning, Hecking, \& Ruck 1983; Thorsson, Prakash, \& Lattimer 1994). In this work, however, we investigate only nuclear matter without strangeness, and we do not consider the pion condensation, the kaon condensation, and the fields of strange hadrons.

The semiclassical energy density (Centelles et al. 1992) of the Lagrangian density is written as

$$
\begin{gathered}
e^{s c}=e_{0}+e_{2}-m \rho+g_{v} V_{0} \rho+e A_{0} \rho_{p}+\frac{1}{3} b \phi_{0}^{3}+\frac{1}{4} c \phi_{0}^{4} \\
+\frac{1}{2}\left[\left(\nabla \phi_{0}\right)^{2}+m_{s}^{2} \phi_{0}^{2}\right]-\frac{1}{2}\left[\left(\nabla V_{0}\right)^{2}+m_{v}^{2} V_{0}^{2}\right]-\frac{1}{2}\left(\nabla A_{0}\right)^{2} \\
-\frac{1}{2}\left[\left(\nabla b_{0}\right)^{2}+m_{\rho}^{2} b_{0}^{2}\right]+\frac{1}{2} g_{\rho} b_{0}\left(\rho_{p}-\rho_{n}\right),
\end{gathered}
$$

where

$$
\begin{gathered}
e_{0}=\left.\sum_{q} \frac{1}{8 \pi^{2}}\left(k_{\mathrm{F}} \epsilon_{\mathrm{F}}^{3}+k_{\mathrm{F}}^{3} \epsilon_{\mathrm{F}}-m^{* 4} \ln \frac{k_{\mathrm{F}}+\epsilon_{\mathrm{F}}}{m^{*}}\right)\right|_{q}, \\
e_{2}=\sum_{q}\left[X_{1 q}\left(\nabla \rho_{q}\right)^{2}+X_{2 q}\left(\nabla \rho_{q} \cdot \nabla m^{*}\right)+X_{3 q}\left(\nabla m^{*}\right)^{2}\right], \\
X_{1 q}=\left.\frac{\pi^{2}}{24 k_{\mathrm{F}}^{3} \epsilon_{\mathrm{F}}^{2}}\left(\epsilon_{\mathrm{F}}+2 k_{\mathrm{F}} \ln \frac{k_{\mathrm{F}}+\epsilon_{\mathrm{F}}}{m^{*}}\right)\right|_{q}, \\
X_{2 q}=\left.\frac{m^{*}}{6 k_{\mathrm{F}} \epsilon_{\mathrm{F}}^{2}} \ln \frac{k_{\mathrm{F}}+\epsilon_{\mathrm{F}}}{m^{*}}\right|_{q}, \\
X_{3 q}=\left.\frac{k_{\mathrm{F}}^{2}}{24 \pi^{2} \epsilon_{\mathrm{F}}^{2}}\left[\frac{\epsilon_{\mathrm{F}}}{k_{\mathrm{F}}}-\left(2+\frac{\epsilon_{\mathrm{F}}^{2}}{k_{\mathrm{F}}^{2}}\right) \ln \frac{k_{\mathrm{F}}+\epsilon_{\mathrm{F}}}{m^{*}}\right]\right|_{q}
\end{gathered}
$$

here $q$ denotes the charge state of each nucleon, $\rho=\rho_{p}+\rho_{n}$ is the baryon number density, $k_{\mathrm{F}}=\left(3 \pi^{2} \rho_{q}\right)^{1 / 3}$ is the Fermi momentum, and $\epsilon_{\mathrm{F}_{q}}=\left(k_{\mathrm{F}_{q}}^{2}+m^{*^{2}}\right)^{1 / 2}$. The $e_{0}$ term is the usual Thomas-Fermi approach, while the $e_{2}$ term is the relativistic correction of the energy density of the order of $\hbar^{2}$.

The semiclassical ground-state density $\rho_{q}$, the meson field, and photon field are obtained by the Euler-Lagrange 
TABLE 1

PARAMETERS OF THE Nonlinear $\sigma \omega$ Models

\begin{tabular}{|c|c|c|}
\hline Parameter & ZM & BB \\
\hline$g_{s}$. & 5.824 & 8.132 \\
\hline$\ldots \ldots$ & 6.417 & 9.598 \\
\hline ...... & 2.746 & 7.520 \\
\hline$b\left(\mathrm{fm}^{-1}\right)$ & 0 & 8.425 \\
\hline$c \ldots \ldots \ldots$ & 0 & 17.398 \\
\hline$m(\mathrm{MeV})$ & 938 & 939 \\
\hline$m_{s}(\mathrm{MeV}) \ldots \ldots \ldots \ldots \ldots \ldots$ & 420 & 500 \\
\hline$m_{v}(\mathrm{MeV}) \ldots \ldots \ldots \ldots \ldots \ldots$ & 783 & 783 \\
\hline$m_{\rho}(\mathrm{MeV}) \ldots \ldots \ldots \ldots \ldots \ldots$ & 763 & 763 \\
\hline
\end{tabular}

equations to the energy density $e^{\text {sc }}$,

$$
\begin{gathered}
\epsilon_{F q}-m+g_{v} V_{0}+\eta_{1} e A_{0}-2 X_{1 q} \nabla^{2} \rho_{q} \\
-X_{2 q} \nabla^{2} m^{*}-\frac{\partial X_{1 q}}{\partial \rho_{q}}\left(\nabla \rho_{q}\right)^{2}-2 \frac{\partial X_{1 q}}{\partial m^{*}}\left(\nabla \rho_{q} \cdot \nabla m^{*}\right) \\
-\left(\frac{\partial X_{2 q}}{\partial m^{*}}-\frac{\partial X_{3 q}}{\partial \rho_{q}}\right)\left(\nabla m^{*}\right)^{2}+\frac{\eta_{2}}{2} g_{\rho} b_{0}=\mu_{q} \\
\left(\nabla^{2}-m_{v}^{2}\right) V_{0}=-g_{v} \rho \\
\left(\nabla^{2}-m_{\rho}^{2}\right) b_{0}=-\frac{1}{2} g_{\rho}\left(\rho_{p}-\rho_{n}\right) \\
\left(\nabla^{2}-m_{s}^{2}\right) \phi_{0}=-\zeta g_{s} \rho_{s}+b \phi_{0}^{2}+c \phi_{0}^{3} \\
\nabla^{2} A_{0}=-e \rho_{p}
\end{gathered}
$$

where $\rho_{s}=\partial e^{\mathrm{sc}} / \partial m^{*}$ is the semiclassical scalar density, $\eta_{1}=$ 0 and $\eta_{2}=-1$ for the neutron, $\eta_{1}=\eta_{2}=1$ for the proton, $\zeta=1$ for the BB model, and $\zeta=\left(\mathrm{m}^{*} / \mathrm{m}\right)^{2}$ for the ZM model, respectively, and $\mu_{q}$ is the chemical potential of nucleon $q$. In solving the above differential equations, $V_{0}, b_{0}$, and $A_{0}$ can be found directly. The scalar field $\phi_{0}$ is solved by iteration until a self-consistent solution is obtained.

From Centelles et al. (1992), we choose the two parameter sets denoted ZM and SRK3M7 listed in Table 1. We have also used these parameter sets to calculate the binding energy, radius, and diffuseness of both ${ }^{40} \mathrm{Ca}$ and ${ }^{208} \mathrm{~Pb}$. The results are well consistent with those of Centelles et al. (1992) (for details, see Yao 1995). As shown in the next section, these parameter sets also yield satisfactory saturation properties (incompressibility, saturation density, and binding energy per nucleon) of symmetric nuclear matter.

In the present study, we consider static infinite matter so that we can obtain simplified equations, in which all the derivative terms in the above Euler-Lagrange equations vanish automatically due to the translational invariance of infinite matter. We will study the properties of nuclear matter and neutron star matter and calculate the structure of neutron stars in the next section.

\section{RESULTS}

\subsection{Properties of Nuclear Matter and Neutron Star Matter}

In Figure 1 we show the energy per nucleon of symmetric nuclear matter calculated in the ZM model and the BB model with SRK3M7. As a comparison, we also display the result based on the relativistic Brueckner-Hartree-Fock (RBHF) theory calculated by Brockmann \& Machleidt (1990). It is obvious that the result of the ZM model resembles that of the RBHF theory, but the difference in the energy per nucleon between the ZM and BB models increases with baryon density at high densities. The satura-

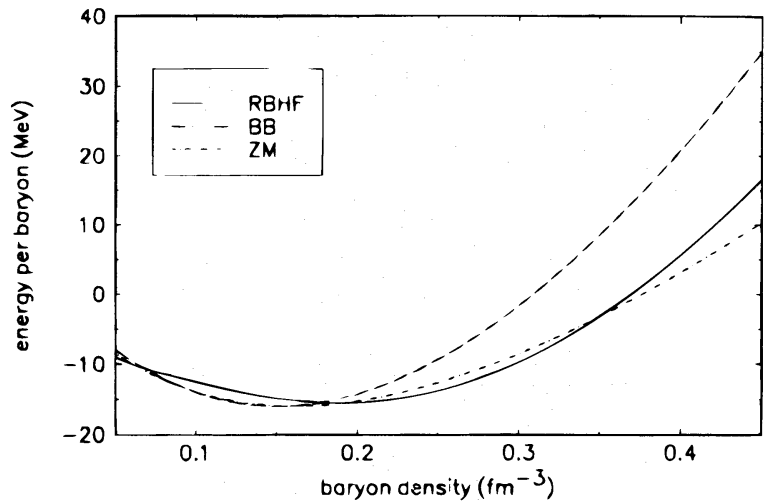

Fig. 1.-The energy per nucleon vs. baryon density for symmetric nuclear matter based on the ZM model, the BB model, and the BruecknerHartree-Fock theory.

tion property of nuclear matter is reproduced by the combination of the attractive scalar $\sigma$ meson and the repulsive vector $\omega$ meson. The energy per nucleon increases linearly with baryon number density at high densities. This is because the repulsive vector $\omega$ meson becomes the dominant contribution to the energy per nucleon at high densities. For the ZM model, the incompressibility, saturation density, and binding energy per nucleon of nuclear matter are $225 \mathrm{MeV}, 0.16 \mathrm{fm}^{-3}$, and $-16.0 \mathrm{MeV}$, respectively, while for the BB model they are $300 \mathrm{MeV}, 0.15 \mathrm{fm}^{-3}$, and $-16.0 \mathrm{MeV}$. These values are very close to those derived from the nuclear experiments.

We apply the nonlinear $\sigma \omega$ models to neutron star matter, which is composed of neutrons, protons, and electrons under the conditions of beta equilibrium and charge neutrality. Here we do not consider the contribution of muons, since they affect the property of the matter and the structure of neutron stars only slightly. Also, we do not consider the contributions of hyperons or other exotic states such as meson condensations or quark matter. Figure 2 shows the energy per nucleon of neutron star matter as a function of baryon density. In Table 2 we list the EOSs based on the ZM and BB models. It is easily observed that our EOS from the ZM model is somewhat softer than that from the BB model.

In Figure 3 we show the proton fraction $Y_{p}$, which is defined as the ratio of the proton density to the baryon density of the neutron star matter, as a function of baryon

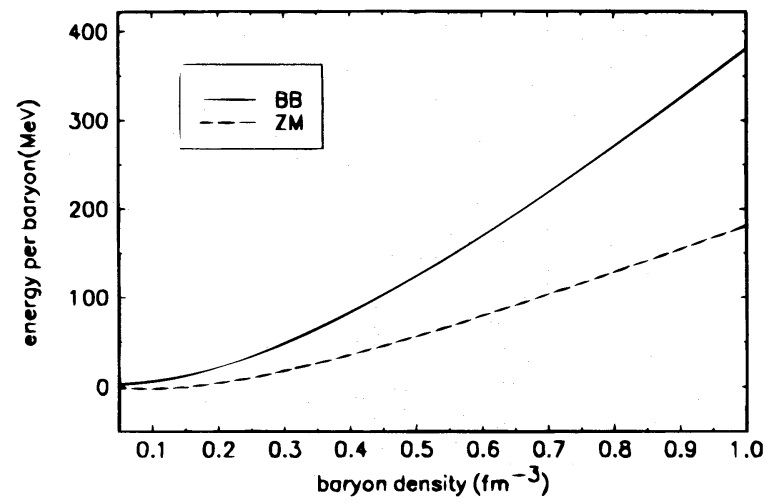

Fig. 2.-The energy per nucleon vs. baryon density for neutron-star matter based on the ZM model and the BB model. 
TABLE 2

Equations of State for Neutron Star Matter Based ON THE NONLINEAR $\sigma \omega$ MODELS

\begin{tabular}{|c|c|c|}
\hline $\begin{array}{l}\text { Baryon Density } \\
\left(\mathrm{fm}^{-3}\right)\end{array}$ & $\begin{array}{c}P_{\mathrm{ZM}} \\
\left(10^{35} \mathrm{dyn} \mathrm{cm}^{-2}\right)\end{array}$ & $\underset{\left(10^{35}{\mathrm{dyn} \mathrm{cm}^{-2}}^{P_{\mathrm{BB}}}\right)}{ }$ \\
\hline 0.14 & 0.0208 & 0.0475 \\
\hline 0.20 & 0.0661 & 0.1381 \\
\hline 0.30 & 0.2206 & 0.4404 \\
\hline 0.40 . & 0.4881 & 0.9662 \\
\hline $0.50 \ldots$ & 0.8726 & 1.7347 \\
\hline $0.60 \ldots \ldots \ldots \ldots$ & 1.3692 & 2.4789 \\
\hline $0.70 \ldots \ldots \ldots \ldots$ & 1.9692 & 4.0028 \\
\hline $0.80 \ldots \ldots \ldots \ldots$ & 2.6640 & 5.4869 \\
\hline $0.90 \ldots$ & 3.4483 & 7.1918 \\
\hline $1.00 \ldots \ldots \ldots \ldots$ & 4.3204 & 9.1115 \\
\hline $1.10 \ldots$ & 5.2849 & 11.244 \\
\hline 1.20 & 6.3491 & 13.593 \\
\hline $1.30 \ldots \ldots \ldots \ldots$ & 7.5237 & 16.164 \\
\hline $1.40 \ldots \ldots \ldots \ldots$ & 8.8184 & 18.965 \\
\hline
\end{tabular}

NoTE.-The subscript " $Z M$ " refers to the $Z M$ model, and the subscript "BB" refers to the BB model.

density. For the BB model, the proton fraction turns out to be large. The fraction increases rapidly as the density increases above the nuclear matter density and reaches a value of 0.22 at $n_{b} \sim 1.0 \mathrm{fm}^{-3}$. But for the BB model, the proton fraction is 0.07 at this density. In $\S 4$ we will discuss the astrophysical implications of these results.

\subsection{Structure of Neutron Stars}

Having the EOS, we can calculate the hydrostatic structure of a neutron star by solving the Tolman-Oppenheimer-

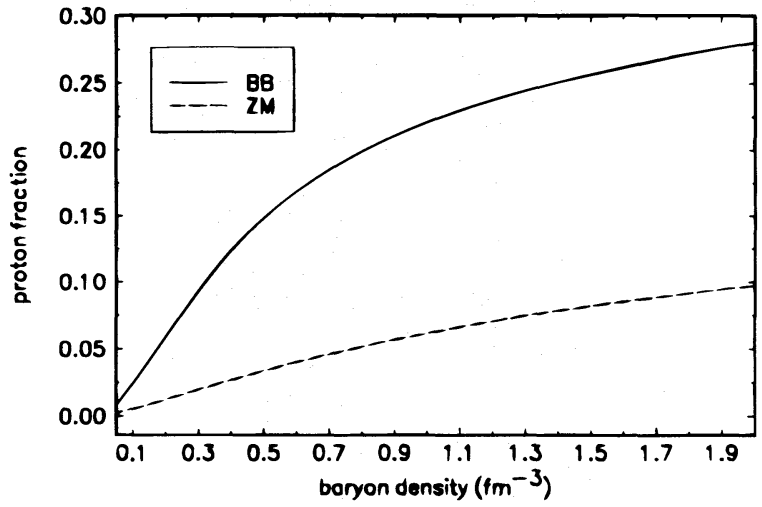

Fig. 3.-Proton fraction vs. baryon density for neutron-star matter based on the ZM and BB models.

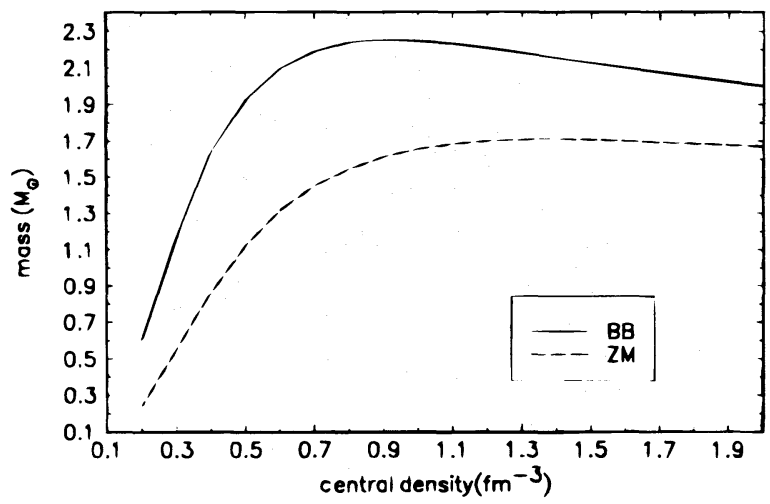

FIG. 4.-Total mass vs. central density for neutron stars based on the $\mathrm{ZM}$ and BB models.

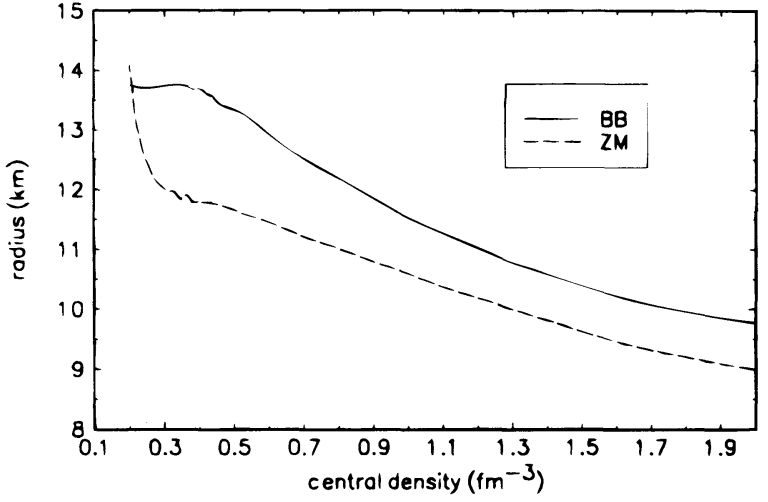

Fig. 5.-Radius vs. central density for neutron stars based on the ZM and BB models.

Volkoff equations:

$$
\frac{d P}{d r}=-\frac{[\rho(r)+P(r)]\left[M(r)+4 \pi r^{3} P(r)\right]}{r^{2}-2 r M(r)},
$$

and

$$
\frac{d M}{d r}=4 \pi \rho(r),
$$

where $P(r)$ and $\rho(r)$ are the pressure and mass density, respectively, and $M(r)$ is the gravitational mass inside a radius $r$. In our calculation, we use the EOS obtained by

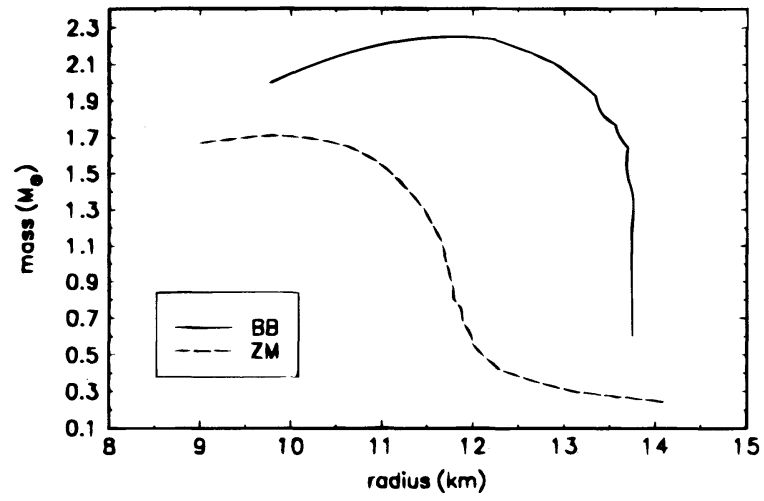

Fig. 6.-Total mass vs. radius for neutron stars based on the ZM and BB models:

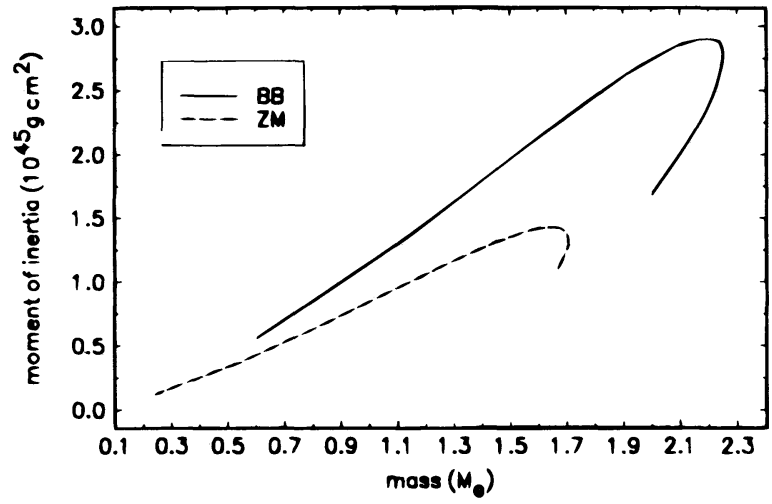

Fig. 7.--Moment of inertia vs. total mass for neutron stars based on the $\mathrm{ZM}$ and $\mathrm{BB}$ models. 


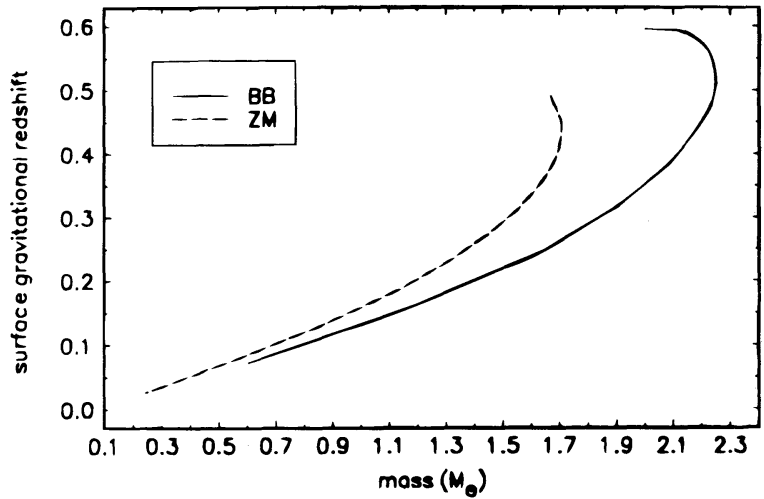

FIG. 8.- Surface gravitational redshift vs. total mass for neutron stars based on the $\mathrm{ZM}$ and $\mathrm{BB}$ models.

Haensel, Zdunik, \& Dobaczewski (1989) for the outer crust of the star, and the EOS derived by Baym, Bethe, \& Pethick (1971) for the inner crust from the neutron-drip density to $0.14 \mathrm{fm}^{-3}$. These EOSs have been expressed rather accurately in the form of polynomials by Bao et al. (1994).

The structure of neutron stars is displayed in Figures 4-10. Figures 4 and 5 show the mass and the radius as a function of the central density, respectively. For the ZM model, the maximum mass of a neutron star is $1.7 M_{\odot}$ at a central density of $n_{c} \approx 1.3 \mathrm{fm}^{-3}$ with a radius $R \approx 10.0 \mathrm{~km}$; for the BB model, the maximum mass is $2.25 M_{\odot}$ at a central density $n_{c} \approx 0.9 \mathrm{fm}^{-3}$ with $R \approx 11.85 \mathrm{~km}$. Figure 6 shows the mass-radius relation for neutron stars. Figures

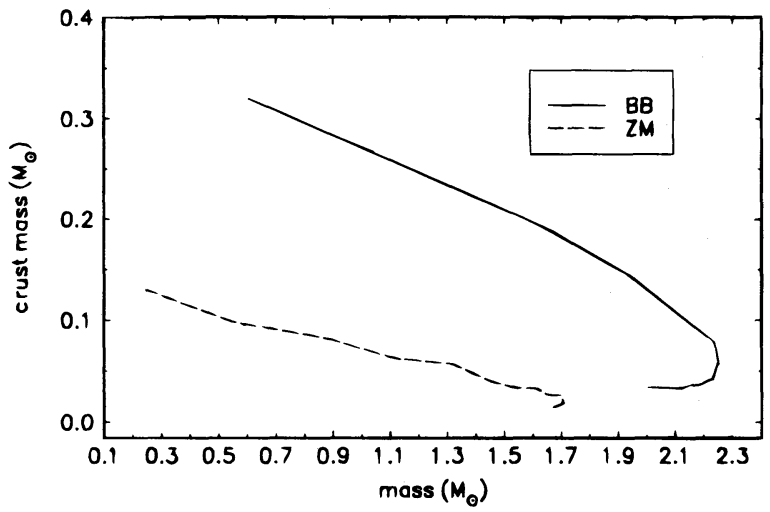

Fig. 9.-Crust (outer plus inner) mass vs. total mass for neutron stars based on the $\mathrm{ZM}$ and $\mathrm{BB}$ models.

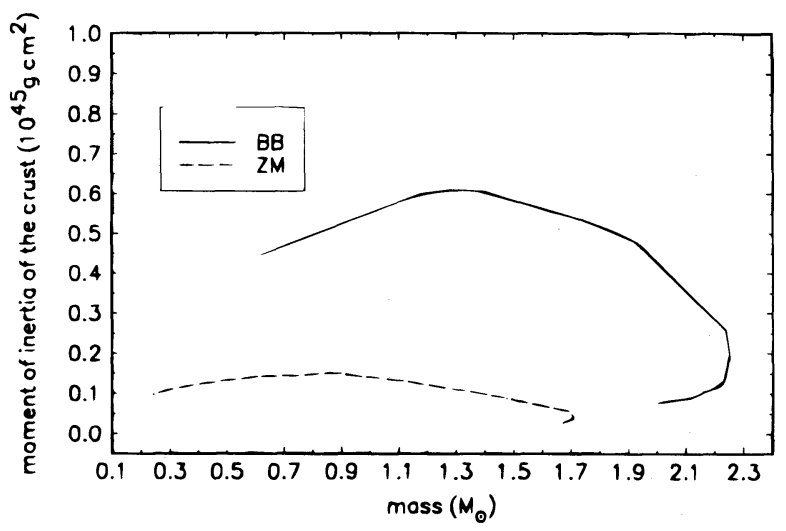

Fig. 10. - Crust (outer plus inner) moment of inertia vs. total mass for neutron stars based on the ZM and BB models.
7-10 show the moment of inertia, surface gravitational redshift, crust mass, and crust moment of inertia as a function of total mass for a neutron star, respectively.

\section{DISCUSSION}

By using the $\mathrm{ZM}$ and $\mathrm{BB}$ models in the relativistic mean field theory, we have calculated the EOS for dense matter and the properties of neutron stars. Now we discuss the astrophysical implications of our results.

First of all, the early observations of binary radio pulsars gave neutron star masses of $1.4 M_{\odot}<M_{\max }<1.85 M_{\odot}$ (Joss \& Rappaport 1984; Taylor \& Weisberg 1989) and the recent observations by Wolszczan (1991) and Thorsett et al. (1993) found $1.1 M_{\odot}<M_{\max }<1.6 M_{\odot}$. These observational results, which seem to eliminate some soft EOSs (Phinney \& Kulkarni 1994), are satisfied by our EOSs. Second, the detections of very fast (in particular, submillisecond) pulsars have stimulated the study of the maximum rotation frequency of uniformly rotating neutron star models. The absolute upper limit on the rotation frequency of a neutron star is the Kepler frequency, at which the gravitational force acting on a mass element at the equator of the star is equal to the centrifugal force. Nonaxisymmetric instabilities driven by gravitational radiation lead to the maximum rotation frequency somewhat lower than the Kepler limit (Friedman \& Ipser 1992, and references therein). Here we do not consider the instabilities, since they are expected to be damped by viscosity of dense matter under typical conditions prevailing in the pulsar interior (Lindblom \& Mendell 1992). Thus, the maximum frequency of uniformly rotating neutron star models is written as

$$
\Omega_{\max }=C\left(\frac{M_{s}}{M_{\odot}}\right)^{1 / 2}\left(\frac{R_{s}}{10 \mathrm{~km}}\right)^{-3 / 2}
$$

where $M_{s}$ and $R_{s}$ are the mass and radius of the maximum allowable mass configuration of nonrotating models. The numerical value of $C$ is $0.77 \times 10^{4} \mathrm{~s}^{-1}$, which is obtained by fitting this formula to numerical values of $\Omega_{\max }$ resulting from realistic calculations for various EOSs (Haensel, Salgado, \& Bonazzola 1995). Hence, for the ZM model $\Omega_{\max }=1.0 \times 10^{4} \mathrm{~s}^{-1}$, and for the BB model $\Omega_{\max }=0.90$ $\times 10^{4} \mathrm{~s}^{-1}$ (see Figs. 4 and 5). Our calculation leads to the minimum rotation period of $0.63 \mathrm{~ms}$ for the $\mathrm{ZM}$ model and $0.70 \mathrm{~ms}$ for the $\mathrm{BB}$ model, which are required by the current observations of millisecond pulsars. Third, prior to the BATSE experiment, many $\gamma$-ray bursts have been observed, $10 \%$ of which have the emission feature (Harding 1991). Katz (1994) suggested that such bursts originate from neutron stars. If the emission line originated from the $e^{+} e^{-} \rightarrow 2 \gamma$ annihilation process at the surface of a neutron star, the observed emission line seems to be redshifted by $\sim 0.2-0.5$ (Liang 1986) or $\sim 0.1-0.3$ (Hartmann 1995). Thus, if we assume that the sources of $\gamma$-ray bursts are standard neutron stars with $1.4 M_{\odot}$, the redshift is about 0.2 for the BB model and about 0.27 for the ZM model. Therefore, the early observations of $\gamma$-ray bursts with emission lines cannot differentiate between these two models. Hopefully, future observations with better accuracy can.

Fourth, pulsar rotation rates have been found that occasionally show sudden increases ("glitches") followed by gradual recoveries that may last days or years. These events are thought to be consequences of angular momen- 
tum transfer between a solid crust, which rotates at the measured pulsar periodicity, and a more rapidly "loose" component, which is believed to be the neutron superfluid in the inner crust (Pines \& Alpar 1985). Sudden braking of the differential rotation between the two components will cause a glitch, and subsequent reestablishment of rotational equilibrium between the two components represents the recovery. Recently Link, Epstein, \& Van Riper (1992) used a model-independent method to analyze the postglitch recovery in four pulsars (Crab, Vela, PSR $0355+54$, and PSR $0525+21$ ) and deduced that the loose component carries at least $0.8 \%$ of the total moment of inertia. This result seems to rule out EOSs that are soft at high densities. A detailed analysis of postglitch recovery based on the vortex creep model (Alpar, Cheng, \& Pines 1989; Alpar et al. 1993) arrived at similar conclusions. For the standard neutron star with $1.4 M_{\odot}$, the ratio of the inner crust to the total moments of inertia is about $8 \%$ for the $\mathrm{ZM}$ model and about $30 \%$ for the BB model (see Figs. 7 and 10). So our EOSs are consistent with the requirement of the observations of glitches.

Finally, we have found that the BB model with SRK3M7 results in a large proton fraction of neutron star matter (see Fig. 3). Similar conclusions have also been drawn by many authors (Boguta 1981; Glendenning 1985; Sumiyoshi \& Toki 1994; Sumiyoshi et al. 1995a, b), who calculated the properties of neutron star matter in the BB model. Thus, the direct Urca process may occur as a main neutrino reaction in a neutron star and would lead to rapid cooling of the star (Lattimer et al. 1991; Page \& Applegate 1992). The direct Urca process is suppressed due to the kinematics when the proton fraction is smaller than 0.11 . So we find that there exists only the modified Urca process in the interior of a neutron star based on the ZM model. One may make a discriminant between the $\mathrm{BB}$ model and the $\mathrm{ZM}$ model through observation of the surface temperature of the stars. Of course, neutron star matter may be in another exotic state such as a pion condensate or a kaon condensate or quark matter (for a recent review, see Pethick 1992). These states could yield copious neutrino emission and rapid cooling of neutron stars. One might also distinguish between these states and the state based on the ZM model by observing the surface radiation of neutron stars.

This work is partly supported by a research grant of the University of Hong Kong. We thank T. Boyce for a critical reading of the manuscript and Siu $\mathbf{M}$. K. for part of the numerical calculation.

\section{REFERENCES}

Alcock, C., Farhi, E., \& Olinto, A. 1986, ApJ, 310, 261

Alpar, M. A., Anderson, P. W., Pines, D., \& Shaham, J. 1984, ApJ, 276, 325

Alpar, M. A., Chau, H. F., Cheng, K. S., \& Pines, D. 1993, ApJ, 409, 345

Alpar, M. A., Cheng, K. S., \& Pines, D. 1989, ApJ, 346, 823

Backer, D., Kulkarni, S. R., Heiles, C., Davies, M. M., \& Goss, W. M. 1982, Nature, 300, 615

Bao, G., Engvik, L., Hjorth-Jensen, M., Osnes, E., \& Ostgaard, E. 1994 Nucl. Phys., A575, 707

Baym, G., Bethe, H. A., \& Pethick, C. J. 1971, Nucl. Phys., A175, 225

Boguta, J. 1981, Phys. Lett., 106B, 255

Boguta, J., \& Bodmer, A. R. 1977, Nucl. Phys., A292, 413 (BB)

Brockmann, R., \& Machleidt, R. 1990, Phys. Rev., C42, 1965

Centelles, M., Viñas, X., Barranco, M., Marcos, S., \& Lombard, R. J. 1992, Nucl. Phys., A537, 486

Centelles, M., Viñas, X., Barranco, M., \& Schuck, P. 1993, Ann. Phys., 221, 165

Chin, S. A. 1977, Ann. Phys., 108, 301

Cook, G. B., Shapiro, S. L., \& Teukolsky, S. A. 1994, ApJ, 424, 823

Ellis, J., Kapusta, J. I., \& Olive, K. A. 1991, Nucl. Phys., B348, 345

Engvik, L., Hjorth-Jensen, M., Osnes, E., Bao, G., \& Ostgaard, E. 1994, Phys. Rev. Lett., 73, 2650

Friedman, J. L., \& Ipser, J. R. 1992, Philos. Trans. R. Soc. London, A340, 391

Friedman, J. L., Ipser, J. R., \& Parker, L. 1989, Phys. Rev. Lett., 62, 617

Glendenning, N. K. 1982, Phys. Lett., B114, 392 . 1985, ApJ, 293, 470

1987a, Z. Phys., A326, 57

1987b, Z. Phys., A327, 295

Glendenning, N. K., Hecking, P., \& Ruck, V. 1983, Ann. Phys., 149, 22

Haensel, P., Salgado, M., \& Bonazzola, S. 1995, A\&A, 296, 745

Haensel, P., Zdunik, J. L., \& Dobaczewski, J. 1989, A\&A, 222, 353

Haensel, P., Zdunik, J. L., \& Schaeffer, R. 1986, A\&A, 160, 121

Harding, A. K. 1991, Phys. Rep., 206, 327

Hartmann, D. 1995, in The Lives of the Neutron Stars, ed. M. A. Alpar, U. Kilziloğlu, \& J. van Paradijs (Dordrecht: Kluwer), 495

Joss, P. C., \& Rappaport, S. A. 1984, ARA\&A, 22, 537

Kapusta, J. I., \& Olive, K. A. 1990, Phys. Rev. Lett., 64, 13

Katz, J. I. 1994, ApJ, 422, 248

Lattimer, J. M., Pethick, C. J., Prakash, M., \& Haensel, P. 1991, Phys. Rev. Lett., 66, 2701
Lattimer, J. M., Prakash, M., Masak, D., \& Yahil, A. 1990, ApJ, 355, 241 Li, G. Q., Machleidt, R., \& Brockmann, R. 1992, Phys. Rev., C46, 2782 Liang, E. P. 1986, ApJ, 304, 682

Lindblom, L., \& Mendell, G. 1992, in Structure and Evolution of Neutron Stars, ed. D. Pines, R. Tamagaki, \& S. Tsuruta (New York: AddisonWesley), 227

Link, B., Epstein, R. I., \& Van Riper, K. A. 1992, Nature, 359, 616

Müther, A., Machleidt, R., \& Brockmann, R. 1990, Phys. Rev., C42, 1981

Ógelman, H. B. 1995, in The Lives of the Neutron Stars, ed. M. A. Alpar,

U. Kilziloğlu, \& J. van Paradijs (Dordrecht: Kluwer), 101

Page, D., \& Applegate, J. H. 1992, ApJ, 394, L17

Pethick, C. J. 1992, Rev. Mod. Phys., 64, 1133

Phinney, E. S., \& Kulkarni, S. R. 1994, ARA\&A, 32, 591

Pines, D., \& Alpar, M. A. 1985, Nature, 316, 27

Prakash, M., Ainsworth, T. L., \& Lattimer, J. M. 1988, Phys. Rev. Lett., 61, 2518

Prakash, M., Cooke, J. R., \& Lattimer, J. M. 1995, Phys. Rev., 52, 661

Serot, B. D., \& Walecka, J. D. 1986, Adv. Nucl. Phys., 16, 1

Sumiyoshi, K., Kuwabara, H., \& Toki, H. 1995a, Nucl. Phys., A581, 725

Sumiyoshi, K., Oyamatsu, K., \& Toki, H. 1995b, RIKEN-AF-NP-198

Sumiyoshi, K., \& Toki, H. 1994, ApJ, 422, 700

Sumiyoshi, K., Toki, H., \& Brockmann, R. 1992, Phys. Lett., B276, 393

Taylor, J. H., Manchester, R. N., \& Lyne, A. G. 1993, ApJS, 88, 529

Taylor, J. H., \& Weisberg, J. M. 1989, ApJ, 345, 908

Thorsett, S. E., Arzoumanian, Z., McKinnon, M. M., \& Taylor, J. H. 1993 ApJ, 405, L29

Thorsson, V., Prakash, M., \& Lattimer, J. M. 1994, Nucl. Phys., A572, 693

Trümper, J., et al. 1978, ApJ, 219, L105

Tsuruta, S. 1995, in The Lives of the Neutron Stars, ed. M. A. Alpar, Ü. Kilziloğlu, \& J. van Paradijs (Dordrecht: Kluwer), 133

Walecka, J. D. 1974, Ann. Phys., 83, 491

Weber, F., \& Weigel, M. K. 1989a, Nucl. Phys., A493, 549

. 1989b, Nucl. Phys., A505, 779

Witten, E. 1984, Phys. Rev., D30, 272

Wolszczan, A. 1991, Nature, 350, 688

Yao, C. C. 1995, Master's thesis, Univ. Hong Kong

Zimanyi, J., \& Moszkowski, S. A. 1990, Phys. Rev., C42, 416 (ZM) 